УДК $511.321+511.31$

\title{
Усиление теоремы Бургейна - Конторовича - III
}

\author{
И. Д. Кан, ${ }^{*}$
}

\begin{abstract}
Аннотация
В настоящей работе доказывается, что в натуральном ряду чисел имеется положительная пропорция знаменателей тех конечных цепных дробей, все неполные частные которых принадлежат алфавиту $\{1,2,3,4,10\}$. Ранее аналогичная теорема была известна лишь для алфавита $\{1,2,3,4,5\}$, либо для алфавитов большей мощности.
\end{abstract}

Библиография: 14 названий.

Ключевые слова и выражения: цепная дробь, континуант, тригонометрическая сумма, гипотеза Зарембы.

\section{1 История вопроса}

Через $\left[d_{1}, d_{2}, \ldots, d_{k}\right]$ обозначают конечную цепную дробь

$$
\left[d_{1}, d_{2}, \ldots, d_{k}\right]=\frac{1}{d_{1}+\ddots_{\cdot+\frac{1}{d_{k}}}}
$$

с натуральными неполными частными $d_{1}, d_{2}, \ldots, d_{k}$ (где $k$ - натуральное), а через $\mathfrak{R}_{\mathcal{A}}$ - множество рациональных чисел $\frac{b}{d}$, представимых конечными цепными дробями с неполными частными из некоторого конечного алфавита $\mathcal{A} \subseteq \mathbb{N}($ всюду далее $|\mathcal{A}| \geqslant 2$ ):

$$
\mathfrak{R}_{\mathcal{A}}=\left\{\frac{b}{d}=\left[d_{1}, d_{2}, \ldots, d_{k}\right] \mid d_{j} \in \mathcal{A} \text { для } j=1, \ldots, k\right\} .
$$

Наконец, через $D_{\mathcal{A}}(N)$ для каждого $N \in \mathbb{N}$ обозначают множество знаменателей $d$ чисел $\frac{b}{d} \in \mathfrak{R}_{\mathcal{A}}$, таких что $d$ не превосходит $N$ :

$$
\mathfrak{D}_{\mathcal{A}}(N)=\left\{d \in \mathbb{N} \mid \exists b:(b, d)=1, \frac{b}{d} \in \mathfrak{R}_{\mathcal{A}}, d \leqslant N\right\}
$$

*Работа выполнена при поддержке РФФИ (грант 12-01-00681-а) 
Гипотеза 1.1. (гипотеза Зарембы [4], 1971). Существует константа A (скорее всего, $A=5$ ), такал что для любого $N \in \mathbb{N}$ для алфавита

$$
\mathcal{A}=1,2, \ldots, A
$$

имеет место равенство $\left|\mathfrak{D}_{\mathcal{A}}(N)\right|=N$.

Подробный обзор результатов, связанных с гипотезой 1.1, можно найти в работах [1, 6]. Отметим лишь, что пргопагандистом и энтузиастом этой темы задолго до 1971 года был профессор Н. М. Коробов. Он, в частности, доказал [7] , что для простого $d$ существует натуральное число $b<d$, такое что $\frac{b}{d} \in \mathfrak{R}_{\mathcal{A}}$ для алфавита $\mathcal{A}$ вида $\sqrt{1.2}$ при $A \leqslant \log d$.

Пусть $\delta_{\mathcal{A}}$ - хаусдорфова размерность множества бесконечных цепных дробей с неполными частными из произвольного конечного алфавита $\mathcal{A}$. Бургейн и Конторович в 2011 году доказали, в частности, следующие две теоремы.

Теорема 1.1. [1, стр.13, теорема 1.25]. Для каждого алфавита $\mathcal{A}$, такого что

$$
\delta_{\mathcal{A}}>\frac{307}{312}=0.9839 \ldots,
$$

справедливо неравенство (“положительная пропориия"):

$$
\left|\mathfrak{D}_{\mathcal{A}}(N)\right|>>N \text {. }
$$

Теорема 1.2. [1, стр.13, теорема 1.27]. Для каждого алвавита $\mathcal{A}$, удовлетворяющего условию (1.3), найдется константа $c=c(\mathcal{A})>0$, такая что

$$
N-\left|\mathfrak{D}_{\mathcal{A}}(N)\right| \ll N^{1-\frac{c}{\log \log N}}
$$

кроме того, каждое число d из отрезка $[1, N]$, за исключением не более чем $N^{1-\frac{c}{\log \log N}}$ чисел, появляется во множестве $\mathfrak{D}_{\mathcal{A}}(N)$ с кратностъю

$$
\left|\left\{b \mid \frac{b}{d} \in \mathfrak{R}_{\mathcal{A}},(b, d)=1\right\}\right|>>_{\epsilon} N^{2 \delta_{\mathcal{A}}-1-\epsilon} .
$$

Результаты Хенсли [2] дают веские основания предполагать, что неравенству (1.3) удовлетворяет алфавит (1.2) при $A=50$ (но не $A=34$ - ввиду результатов Дженкинсона [3] ).

Автор настоящей статьи совместно с Д. А. Фроленковым ([8] - [11]) усилили первую из упомянутых теорем Бургейна - Конторовича. Усиление состояло в понижении достаточной нижней оценки $\delta_{\mathcal{A}}$ вида $(1.3)$ при сохранении итогового неравенства (1.4). Так, в работе [1] неравенство (1.4) было доказано лишь при условии

$$
\delta_{\mathcal{A}}>\frac{5}{6}=0.8333 \ldots
$$

соответствующем алфавиту (1.2) при $A=5$, но аналогичное обобщение теоремы 1.2 выведено не было.

Недавно (2013 г.), объединяя методы работ [1] и [11], С. Хуанг [13] доказал, что свойства (1.5) и (1.6) при выполнении неравенства (1.7) также справедливы. Работа [13] 
содержит два примечательных достижения. Одно из них состоит в упрощении метода Бургейна - Конторовича в части вывода неравенств 1.5) и 1.6. Другое, неменее важное, - может быть сформулировано в виде тезиса:

какова бы ни была нижняя грань хаусдорбовой размерности $\delta_{\mathcal{A}}$, позволяющая вывести неравенство (1.4) в рамках рассматриваемого метода, та же самая оченка $\delta_{\mathcal{A}}$ позволяет получать утверждения (1.5) и (1.6) аналогичным образом, используя аргументы работы [13].

\section{2 Основные результаты работы}

Основной результат настоящей статьи - следующий.

Теорема 2.1. Для произвольного алфавита $\mathcal{A}$, такого что

$$
\delta_{\mathcal{A}}>\frac{4}{5}=0.8
$$

имеет место неравенство $\left|\mathfrak{D}_{\mathcal{A}}(N)\right|>>N$.

Применение методов Хуанга позволяет теперь вывести следующую теорему.

Теорема 2.2. Для произвольного алфавита $\mathcal{A}$, удовлетворяющего неравенству (2.1), имеют место оченки (1.5) и (1.6).

Замечание 2.1. Согласно результатам работь Дюенкинсона [3] , неравенству (2.1) удовлетворяют все алфавиты вида

$$
\mathcal{A}=\{1,2,3,4, n\},
$$

где число п может принимать любое из значений 6, 7,8,9,10.

\section{3 Благодарности.}

Автор благодарит профессора Н. Г. Мощевитина за постановку задачи и неоднократное обсуждение темы статьи. Также автор благодарен И. Д. Шкредову и И. С. Резвяковой за вопросы и комментарии во время докладов автора. Кроме того, автор весьма благодарен Д. А. Фроленкову за многие полезные советы - в частности, совет использовать методы работы [13].

\section{4 Обозначения.}

Всюду далее $\epsilon_{0} \in\left(0, \frac{1}{2500}\right)$ - произвольно малая положительная константа, участвующая в построении ансамбля $\Omega_{N}$. Знак Виноградова $f(N) \ll g(N)$ для двух произвольных функций $f(N)$ и $g(N)$ обозначает существование константы $C$, зависящей только от $\mathcal{A}$ и $\epsilon_{0}$, такой что $|f(N)| \leqslant C g(N)$. Если при этом $C=C(\epsilon)$ для произвольного $\epsilon>0$, то используется обозначение $f(N) \ll_{\epsilon} g(N, \epsilon)$. Также используется традиционное обозначение $e(x)=\exp (2 \pi i x)$. Наибольший общий делитель двух целых чисел $a$ и $b$ обозначается через $(a, b)$. Если $P$ - утверждение, то $\mathbf{1}_{\{P\}}=1$, когда $P$ истинно, и $\mathbf{1}_{\{P\}}=0$, когда $P$ ложно. Мощность конечного множества $S$ обозначается через $|S|$. Для действительного 
числа $\alpha$ через $[\alpha],\{\alpha\}$ и $\|\alpha\|$ обозначаются, соответственно, целая часть от $\alpha$, дробная доля $\alpha$ и расстояние от $\alpha$ ближайшего целого:

$$
[\alpha]=\max \{z \in \mathbb{Z} \mid z \leqslant \alpha\}, \quad\{\alpha\}=\alpha-[\alpha], \quad\|\alpha\|=\min \{|z-\alpha| \mid z \in \mathbb{Z}\} .
$$

Кроме того, если $g$ - матрица, то $\|g\|$ - ее норма (определенная ниже в параграфе 5).

\section{5 Континуанты и матрицы.}

Континуантом натуральных чисел $d_{1}, d_{2}, \ldots, d_{k}$ называется число $\left\langle d_{1}, d_{2}, \ldots, d_{k}\right\rangle$, равное знаменателю цепной дроби (1.1), несократимому с ее числителем (в том числе, континуант пустой последовательности считается равным одному). Если последовательность $d_{1}, d_{2}, \ldots, d_{k}$ обозначена через $D$, то через $\stackrel{\leftarrow}{D}$ обозначают последовательность $d_{k}, d_{k-1}, \ldots, d_{1}$, а через $D^{-}$и $D_{-}$- последовательности $d_{1}, d_{2}, \ldots, d_{k-1}$ и $d_{2}, d_{3}, \ldots, d_{k}$, соответственно. Хорошо известно (например, [5]), что для произвольных конечных последовательностей $D, X$ выполнено неравенство

$$
\langle D\rangle\langle X\rangle \leqslant\langle D, X\rangle \leqslant 2\langle D\rangle\langle X\rangle,
$$

следующее из равенства

$$
\langle D, X\rangle=(1+[\overleftarrow{D}][X])\langle D\rangle\langle X\rangle
$$

и что для матрицы

$$
g=\left(\begin{array}{ll}
a & b \\
c & d
\end{array}\right)=\left(\begin{array}{ll}
0 & 1 \\
1 & d_{1}
\end{array}\right)\left(\begin{array}{ll}
0 & 1 \\
1 & d_{2}
\end{array}\right) \ldots\left(\begin{array}{ll}
0 & 1 \\
1 & d_{k}
\end{array}\right)
$$

выполнены соотношения $(b, d)=(c, d)=1, a<c<d, a<b<d$,

$$
\frac{b}{d}=\frac{\left\langle D_{-}\right\rangle}{\langle D\rangle}=\left[d_{1}, d_{2}, \ldots, d_{k}\right]=[D], \frac{c}{d}=\frac{\left\langle D^{-}\right\rangle}{\langle D\rangle}=\left[d_{k}, d_{k-1}, \ldots, d_{1}\right]=[\overleftarrow{D}]
$$

Следовательно, для нормы такой матрицы $g$ имеет место равенство

$$
\|g\|=\max \{|a|,|b|,|c|,|d|\}=d=\left\langle d_{1}, d_{2}, \ldots, d_{k}\right\rangle .
$$

Лемма 5.1. (сходное утверждение имеется в [12]). Пусть $D, T, W-$ конечные последовательности чисел из алфавита (1.2), в том числе $T$ и $W$ - непустые и имеют различие в своих первых элементах и равную длину. Тогда справедливо неравенство

$$
|[D, T]-[D, W]| \geqslant \frac{1}{(2 A)^{4}\langle D\rangle^{2}}
$$

Доказательство. Прежде всего, для любой непустой конечной последовательности $V$ в алфавите 1.2 имеет место оценка

$$
\frac{1}{2 A} \leqslant[A, 1] \leqslant[V] \leqslant 1
$$


Пусть, для начала, последовательность $D$ не пуста. Тогда, если $X$ - любая из последовательностей $T$ или $W$, то, используя равенства (5.2) и (5.4), получаем:

$$
[D, X]=\frac{\left\langle(D, X)_{-}\right\rangle}{\langle D, X\rangle}=\frac{\left(1+\left[(\overleftarrow{D})^{-}\right][X]\right)\left\langle D_{-}\right\rangle\langle X\rangle}{(1+[\overleftarrow{D}][X])\langle D\rangle\langle X\rangle}=[D] \frac{1+[X]\left[(\overleftarrow{D})^{-}\right]}{1+[X][\overleftarrow{D}]}
$$

Следовательно, обозначая левую часть неравенства (5.6) через $\sigma$, получаем:

$$
\sigma=[D] \frac{\left|\left([\stackrel{\leftarrow}{D}]-\left[(\overleftarrow{D})^{-}\right]\right)([W]-[T])\right|}{(1+[T][\overleftarrow{D}])(1+[W][\overleftarrow{D}])} \geqslant \frac{\left|\left([\overleftarrow{D}]-\left[(\overleftarrow{D})^{-}\right]\right)([W]-[T])\right|}{8 A}
$$

в виду неравенства (5.7), примененного к каждому из случаев $V=D, \overleftarrow{D}, T, W$. Так как, кроме того, имеют место оценки

$$
\begin{gathered}
\left|[\overleftarrow{D}]-\left[(\overleftarrow{D})^{-}\right]\right|=\frac{1}{\langle D\rangle\left\langle D_{-}\right\rangle} \geqslant \frac{1}{\langle D\rangle^{2}} \\
|[W]-[T]| \geqslant[A-1,1]-[A, A, 1] \geqslant \frac{1}{2 A^{3}}
\end{gathered}
$$

то, подставляя их в (5.8), получаем (5.6).

Наконец, если последовательность $D$ пуста, то нужное неравенство (5.6) следует непосредственно из (5.9). Лемма доказана.

\section{6 Основные свойства ансамбля $\Omega_{N}$.}

Через $\Gamma_{\mathcal{A}}$ обозначают мультипликативную полугруппу $\Gamma_{\mathcal{A}} \subseteq S L(2, \mathbb{Z})$ с единицей $E=\left(\begin{array}{ll}1 & 0 \\ 0 & 1\end{array}\right)$, порожденную попарными матричными произведениями

$$
\left(\begin{array}{ll}
0 & 1 \\
1 & a
\end{array}\right)\left(\begin{array}{ll}
0 & 1 \\
1 & b
\end{array}\right)=\left(\begin{array}{cc}
1 & b \\
a & a b+1
\end{array}\right)
$$

где $a, b \in \mathcal{A}$. Обозначим через $V_{\mathcal{A}}$ множество слов четной длины $(=$ состоящих из четного количества букв) в алфавите $\mathcal{A}$. Далее всюду будем использовать взаимную однозначность отображения $\mathcal{B}: V_{\mathcal{A}} \rightarrow \Gamma_{\mathcal{A}}$, определенного формулой

$$
\mathcal{B}\left(d_{1}, d_{2}, \ldots, d_{2 k}\right)=\left(\begin{array}{cc}
0 & 1 \\
1 & d_{1}
\end{array}\right)\left(\begin{array}{cc}
0 & 1 \\
1 & d_{2}
\end{array}\right) \ldots\left(\begin{array}{cc}
0 & 1 \\
1 & d_{2 k}
\end{array}\right)
$$

Напомним, что подмножество $\Theta$ матриц $g \in \Gamma_{\mathcal{A}}$ называется предансамблем [10, параграф 8], если для любых двух матрищ $g_{1}, g_{2} \in \Gamma_{\mathcal{A}}$ и любого положительного числа $\epsilon$ выполнены соотношения $\left\|g_{1}\right\| \ll\left\|g_{2}\right\| \ll\left\|g_{1}\right\|$ и $|\Theta|>>_{\epsilon}\left\|g_{1}\right\|^{2 \delta_{\mathcal{A}}-\epsilon}$. По произвольному достаточно большому числу $N$ и по малому параметру $\epsilon_{0} \in\left(0, \frac{1}{2500}\right)$ в [10] была построена конечная последовательность

$$
\left\{N_{-J-1}, N_{-J}, \ldots, N_{-1}, N_{0}, N_{1}, \ldots, N_{J+1}\right\},
$$


где $N_{J+1}=N, J \ll \log \log N$,

$$
N_{j}= \begin{cases}N^{\frac{1}{2-\epsilon_{0}}\left(1-\epsilon_{0}\right)^{1-j}}, & \text { если }-1-J \leqslant j \leqslant 1 ; \\ N^{1-\frac{1}{2-\epsilon_{0}}\left(1-\epsilon_{0}\right)^{j}}, & \text { если } 0 \leqslant j \leqslant J,\end{cases}
$$

имеющая такое свойство [10, лемма 9.1]: при $-J-1 \leqslant m \leqslant J-1$ выполнены неравенства

$$
N_{j-J-1} \geqslant N_{j-J}^{1-\epsilon_{0}}, \quad \frac{N}{N_{j-J}} \geqslant\left(\frac{N}{N_{j-J-1}}\right)^{1-\epsilon_{0}} .
$$

Также в [10] по последовательности $\left\{N_{j}\right\}$ было построено специальное множество матриц - ансамбль $\Omega_{N} \subseteq\left\{g \in \Gamma_{\mathcal{A}} \mid\|g\| \leqslant 1,02 N\right\}$ (терминология и существо дела - из [1]),

$$
\Omega_{N}=\Xi_{1} \Xi_{2} \ldots \Xi_{2 J} \Xi_{2 J+1},
$$

где $J$ - из (6.2), а все множества $\Xi_{j}$ - предансамбли.

Отметим, что для доказательства неравенств (1.5) и (1.6) Хуанг в работе [13] использовал несколько иной вариант ансамбля $\Omega_{N}$, который отличается, в основном, тем, что среди множеств-множителей в правой части равенства (6.5) присутствует некоторое специальное множество матриц $\chi$ (впервые построенное в [1] и перенесенное Хуангом из начала последовательности множеств, сходной с 6.5), в “середину" той же последовательности). Строго говоря, аналогичное видоизменение ансамбля требуется и в настоящей работе - для тех же целей. Но поскольку такой подход приводит к существенному усложнению выкладок, будем для краткости всегда полагать, что ансамбль задан равенством 6.5).

Напомним основные свойства ансамбля.

Лемма 6.1. [10, лемма 11.2]. Для любого набора матрии,

$$
\xi_{1} \in \Xi_{1}, \xi_{2} \in \Xi_{2}, \ldots, \xi_{2 J+1} \in \Xi_{2 J+1},
$$

для любого $j$ из интервала $1 \leqslant j \leqslant 2 J+1$ выполнено неравенство

$$
\frac{1}{70 A^{2}} N_{j-J} \leqslant\left\|\xi_{1} \xi_{2} \ldots \xi_{j}\right\| \leqslant 1,01 N_{j-J}
$$

а для каждого $j$ из интервала $0 \leqslant j \leqslant 2 J$ имеет место аналогичное неравенство

$$
\frac{1}{150 A^{2}} \frac{N}{N_{j-J}} \leqslant\left\|\xi_{j+1} \xi_{j+2} \ldots \xi_{2 J+1}\right\| \leqslant 73 A^{2} \frac{N}{N_{j-J}} .
$$

Следствие 6.1. Если числа ј и U связаны соотношением

$$
N_{j-J-1} \leqslant U<N_{j-J},
$$

то в обозначениях предыдущей леммы выполнены неравенства

$$
\begin{gathered}
\frac{1}{70 A^{2}} U \leqslant\left\|\xi_{1} \xi_{2} \ldots \xi_{j}\right\| \leqslant 1,01 U^{1+2 \epsilon_{0}}, \\
\frac{1}{150 A^{2}} \max \left\{\frac{N}{U^{1+2 \epsilon_{0}}},\left(\frac{N}{U}\right)^{1-\epsilon_{0}}\right\} \leqslant\left\|\xi_{j+1} \xi_{j+2} \ldots \xi_{2 J+1}\right\| \leqslant 73 A^{2} \frac{N}{U} .
\end{gathered}
$$


Доказательство. Из неравенств (6.4) и (6.8) следует двусторонняя оценка числа $N_{j-J}$ :

$$
U \leqslant N_{j-J} \leqslant \min \left\{U^{1+2 \epsilon_{0}}, U^{1-\epsilon_{0}} N^{\epsilon_{0}}\right\} .
$$

Подставляя ее в (6.6) и (6.7), получаем 6.9) и (6.10). Следствие доказано.

Пусть $M^{(1)}>1$ - некоторое действительное число. Определим числа

$$
\begin{gathered}
H=H_{1}\left(M^{(1)}\right)=1,01\left(M^{(1)}\right)^{1+2 \epsilon_{0}}, \\
Q_{1}=\exp \left(A^{4} \epsilon_{0}^{-5}\right)
\end{gathered}
$$

и интервал

$$
I_{N}=\left[Q_{1}, N\right] .
$$

Теорема 6.1. (см. [11, теорема 4.2]). Пусть числа

$$
M^{(1)} \in I_{N}, \quad M^{(2)}, M^{(4)} \in I_{N} \bigcup\{1\}
$$

удовлетворяют неравенству

$$
M^{(1)} M^{(2)} M^{(4)} \leqslant N
$$

Тогда найдутся целье числа - индексы $j_{1}, j_{2}, j_{3}$, такие что

$$
1=j_{0} \leqslant j_{1} \leqslant j_{2} \leqslant j_{3} \leqslant j_{4}=2 J+2
$$

и для $k=1,2,3,4$ в обозначения

$$
\Omega^{(k)}=\Xi_{j_{k-1}} \Xi_{j_{k-1}+1} \Xi_{j_{k-1}+2} \ldots \Xi_{j_{k}-1}
$$

имеют место соотношения 6.17) - 6.18) (ниже), а для любых четырех матрии,

$$
g_{1} \in \Omega^{(1)}, g_{2} \in \Omega^{(2)}, g_{3} \in \Omega^{(3)}, g_{4} \in \Omega^{(4)}
$$

выполняются неравенства (6.19) - 6.23) (ниже); в частности, если $M^{(2)}=1$ или $M^{(4)}=1$, то $\Omega^{(2)}=\{E\}$ или $\Omega^{(4)}=\{E\}$, соответственно.

Где:

- произведение в (6.15) по пустому множеству индексов (при $j_{k-1}=j_{k}$ ) считается равным $\{E\}, E-$ единичная матрица размера $2 \times 2$;

$$
\begin{gathered}
\Omega_{N}=\Omega^{(1)} \Omega^{(2)} \Omega^{(3)} \Omega^{(4)} \\
\left|\Omega^{(1)} \Omega^{(2)}\right|>>\left(M^{(1)} M^{(2)}\right)^{2 \delta_{\mathcal{A}}-\epsilon_{0}},\left|\Omega^{(4)}\right|>>\left(M^{(4)}\right)^{2 \delta_{\mathcal{A}}-2 \epsilon_{0}}, \\
\frac{M^{(1)}}{70 A^{2}} \leqslant\left\|g_{1}\right\| \leqslant H \\
\frac{N}{150 A^{2} H} \leqslant\left\|g_{2} g_{3} g_{4}\right\| \leqslant 73 A^{2} \frac{N}{M^{(1)}} \\
\left\|g_{2}\right\| \leqslant 73 A^{2} M^{(2)}\left(M^{(1)} M^{(2)}\right)^{2 \epsilon_{0}}
\end{gathered}
$$




$$
\begin{gathered}
\frac{\left(M^{(4)}\right)^{1-\epsilon_{0}}}{150 A^{2}} \leqslant\left\|g_{4}\right\| \leqslant 73 A^{2} M^{(4)}, \\
\frac{M^{(1)} M^{(2)}}{70 A^{2}} \leqslant\left\|g_{1} g_{2}\right\| \leqslant 1,01\left(M^{(1)} M^{(2)}\right)^{1+2 \epsilon_{0}} .
\end{gathered}
$$

Доказательство. Положим

$$
U^{(1)}=M^{(1)}, U^{(2)}=M^{(1)} M^{(2)}, U^{(3)}=\frac{N}{M^{(4)}}, U^{(4)}=N .
$$

Тогда, ввиду (6.13), имеет место цепочка неравенств:

$$
U^{(1)} \leqslant U^{(2)} \leqslant U^{(3)} \leqslant U^{(4)} .
$$

Поэтому, если для $k=1,2,3$ числа $j_{k}$ определить неравенствами

$$
N_{j_{k}-J-1} \leqslant U^{(k)}<N_{j_{k}-J}
$$

то, ввиду 6.24 и монотонности последовательности $\left\{N_{j}\right\}$, имеют место неравенства 6.14. Следовательно, из 6.5 и 6.15) можно вывести равенство (6.17). Далее, условия следствия 6.1 выполнены при $k=1,2,3$ для $U=U^{(k)}$ и $j=j_{k}$, для которых, поэтому, имеют место оценки (6.9) и 6.10). Первая из них при подстановках $U=U^{(1)}=M^{(1)}$ и $U=U^{(2)}=M^{(1)} M^{(2)}$ дает неравенства 6.19 и (6.23), соответственно. Аналогично, вторая из оценок следствия 6.1 дает неравенства 6.20) и 6.22 при подстановках $U=$ $U^{(1)}=M^{(1)}$ и $U=U^{(3)}=\frac{N}{M^{(4)}}$, соответственно. Комбинация доказанных оценок $\sqrt{6.19}$ и 6.23 дает неравенство 6.21), так как, в виду нижней оценки в (5.1) и равенства (5.5), выполняется оценка

$$
\left\|g_{2}\right\| \leqslant \frac{\left\|g_{1} g_{2}\right\|}{\left\|g_{1}\right\|}
$$

Наконец, неравенства 6.18) выводятся с помощью теоремы [10, теорема 12.4], исходя из нижних оценок в доказанных неравенствах 6.22 и (6.23). Теорема доказана.

В условиях последней теоремы положим:

$$
\Omega=\Omega^{(2)} \Omega^{(3)} \Omega^{(4)}=\Xi_{j_{1}} \Xi_{j_{1}+1} \ldots \Xi_{2 J+1} .
$$

Договоримся использовать следующие обозначения: если $g-$ матрица размера $2 \times 2$, то $\widetilde{g}$ - вектор-столбец, получающийся из матрицы $g$ домножением на $\left(\begin{array}{l}0 \\ 1\end{array}\right)$ :

$$
\widetilde{g}=g\left(\begin{array}{l}
0 \\
1
\end{array}\right)
$$

Кроме того, если $X-$ некоторое множество матриц $g$ размера $2 \times 2$, то $\widetilde{X}-$ множество соответствующих им вектор-столбцов $\widetilde{g}$. Далее, выберем произвольный элемент $g_{3} \in \Omega^{(3)}$ и положим

$$
\Omega\left(g_{3}\right)=\Omega^{(2)}\left\{g_{3}\right\} \Omega^{(4)}=\left\{g=g_{2} g_{3} g_{4} \mid g_{2} \in \Omega^{(2)}, g_{4} \in \Omega^{(4)}\right\} .
$$

Тогда, если $\widetilde{g} \in \widetilde{\Omega}\left(g_{3}\right)$, то $\widetilde{g}-$ вектор-столбец. Для двух произвольных векторов из $\widetilde{\Omega}\left(g_{3}\right)$ введем обозначения:

$$
\widetilde{g}^{(1)}=g_{2}{ }^{(1)} g_{3} g_{4}{ }^{(1)}\left(\begin{array}{l}
0 \\
1
\end{array}\right)=\left(\begin{array}{l}
x_{1} \\
x_{2}
\end{array}\right), \widetilde{g}^{(2)}=g_{2}{ }^{(2)} g_{3} g_{4}{ }^{(2)}\left(\begin{array}{l}
0 \\
1
\end{array}\right)=\left(\begin{array}{l}
y_{1} \\
y_{2}
\end{array}\right) .
$$


Следствие 6.2. Пусть выполнены условия последней теоремы. Тогда, во-первых, имеет место неравенство

$$
\max _{g \in \Omega}\|g\| \leqslant 11000 A^{4} \min _{g \in \Omega}\|g\|
$$

во-вторых, для векторов (6.27) для кажсдого $i \in\{1,2\}$ выполняются неравенства:

$$
\begin{gathered}
\max \left\{x_{i}, y_{i}\right\} \leqslant 22000 A^{5} x_{i}, \\
\frac{N}{2 H} \leqslant 150 A^{3} x_{i} .
\end{gathered}
$$

Доказательство. Согласно лемме 6.1. в обозначениях 6.25 при $j=j_{1}$ имеет место неравенство (6.7), в котором верхняя оценка отличается от нижней не более, чем в $11000 A^{4}$ раз. Этим обстоятельством доказаны как неравенство 6.28), так и оценка 6.29 при $i=2$ ввиду (5.5). Далее, из (5.1) следует, что $x_{1} \leqslant x_{2} \leqslant 2 A x_{1}$. Поэтому, ввиду доказанного неравенства (6.28), имеет место цепочка оценок:

$$
\max \left\{x_{1}, y_{1}\right\} \leqslant \max \left\{x_{2}, y_{2}\right\} \leqslant 11000 A^{4} \min \left\{x_{2}, y_{2}\right\} \leqslant 11000 A^{4} x_{2} \leqslant 22000 A^{5} x_{1},
$$

так что неравенство (6.29) доказано и при $i=1$. Наконец, если в нижней из оценок 6.20 положить $g_{2}=g_{2}^{(1)}, g_{4}=g_{4}^{(1)}$ и учесть обозначения 6.27, то получим:

$$
\frac{N}{2 H} \leqslant 75 A^{2} x_{2} \leqslant 150 A^{3} x_{1} \leqslant 150 A^{3} x_{i}
$$

для каждого $i \in\{1,2\}$, и неравенство 6.30 также доказано. Следствие доказано.

\section{7 Достаточные условия выполнения оценок (1.4) - (1.6).}

Согласно методу Бургейна - Конторовича [1], оценка (1.4) следует из неравенства

$$
\int_{0}^{1}\left|S_{N}(\Theta)\right|^{2} d \Theta \ll \frac{\left|\Omega_{N}\right|^{2}}{N}
$$

где

$$
S_{N}(\Theta)=\sum_{g \in \Omega_{N}} e(\Theta\|g\|)=\sum_{g \in \Omega_{N}} e\left((0,1) g\left(\begin{array}{l}
0 \\
1
\end{array}\right) \Theta\right)=\sum_{g \in \Omega_{N}} e((0,1) \widetilde{g} \Theta)
$$

- тригонометрическая сумма.

Применяя теорему Дирихле [14, лемма 2.1, стр.17], для каждого $\Theta \in[0,1)$ найдем целые числа $a$ и $q$ и действительное число $K$, такие что

$$
\Theta=\left\{\frac{a}{q}+\frac{K}{N}\right\},(a, q)=1,0 \leqslant a<q \leqslant \frac{N^{\frac{1}{2}}}{Q_{1}}, \quad|K| \leqslant \frac{Q_{1} N^{\frac{1}{2}}}{q}
$$

(где фигурные скобки обозначают дробную долю, а величина $Q_{1}$ была определена в (6.12) ), при чем равенство $a=0$ возможно только при $q=1$. Далее, представим число $K$ в виде

$$
K=\frac{1}{2} l+\lambda, \lambda \in\left(-\frac{1}{4}, \frac{1}{4}\right]
$$


где $l$ - какое-либо целое число, тогда $|l| \leqslant 2|K|+1$, поэтому, ввиду 7.3 , имеет место оценка

$$
|l| \leqslant \frac{3}{q} Q_{1} N^{\frac{1}{2}}
$$

Для произвольного неотрицательного параметра $Q=Q(N)$ определим величину $\Sigma_{N}(Q)$ :

$$
\Sigma_{N}(Q)=\int_{-\frac{1}{4}}^{\frac{1}{4}} \sum_{0 \leqslant a<q \leqslant \frac{1}{Q_{1}} N^{\frac{1}{2}}}^{*} \sum_{|l| \leqslant \frac{3}{q} Q_{1} N^{\frac{1}{2}}} \mathbf{1}_{\{\max \{q,|l|\} \geqslant Q\}}\left|S_{N}\left(\frac{a}{q}+\frac{l}{2 N}+\frac{\lambda}{N}\right)\right|^{2} d \lambda,
$$

где сумма $\sum^{*}$ берется по парам взаимно простых чисел $a$ и $q$.

Лемма 7.1. Неравенство (1.4) имеет место, если найдется хотя бы одно действительное число $\epsilon_{0} \in\left(0, \frac{1}{2500}\right)$, такое что выполнено неравенство

$$
\Sigma_{N}(0) \ll\left|\Omega_{N}\right|^{2} .
$$

Доказательство. Положим $Q=0$ и оценим сверху интеграл из (7.1) интегралом из (7.6), деленным на $N$. Для этого достаточно представить $\Theta$ в виде (7.3), где число $K$ взято из (7.4), разбить отрезок интегрирования в (7.1) на ряд отрезков, соответствующих значениям параметров $a, q, l$, и в получившихся интегралах сделать линейную замену $\lambda=N\left(\Theta-\frac{a}{q}-\frac{l}{2 N}\right)$. Лемма доказана.

Замечание 7.1. Согласно результатам работы [13], неравенства (1.5) и (1.6] имеют место, если найдется хотя бы одно действительное число $\epsilon_{0} \in\left(0, \frac{1}{2500}\right)$, такое что для некоторой константы $C=C(\mathcal{A})>0$ для любой сколь угодно медленно стремящейся к бесконечности величины $Q=Q(N)$ выполнено неравенство

$$
\Sigma_{N}(Q) \ll\left|\Omega_{N}\right|^{2} Q^{-C} .
$$

Напомним, что $Q_{1}$ было определено в 6.12, положим $Q_{0}=0$ и определим последовательность $\left\{Q_{j}\right\}$ для $j$ от нуля до бесконечности:

$$
\left\{Q_{j}\right\}_{j=0}^{\infty}=\left\{0, Q_{1}, Q_{1}^{2}, Q_{1}^{3}, \ldots, Q_{1}^{j}, \ldots\right\} .
$$

При фиксированном $\lambda \in\left(-\frac{1}{4}, \frac{1}{4}\right]$ для целых $\alpha, \beta \geqslant 0$ положим:

$$
P_{\alpha, \beta}^{(\lambda)}=\left\{\Theta=\frac{a}{q}+\frac{l}{2 N}+\frac{\lambda}{N} \mid(a, q)=1,0 \leqslant a<q, \text { выполнены 7.11) и } 7.12\right\},
$$

где

$$
\begin{gathered}
Q_{\alpha} \leqslant q \leqslant \min \left\{\frac{1}{Q_{1}} N^{\frac{1}{2}}, Q_{\alpha+1}\right\}, \\
Q_{\beta} \leqslant|l| \leqslant \min \left\{\frac{3}{q} Q_{1} N^{\frac{1}{2}}, Q_{\beta+1}\right\} .
\end{gathered}
$$

Замечание 7.2. Заметим, что для чисел $Q_{\alpha+1}, Q_{\beta+1}$, при $\alpha, \beta \geqslant 0$ для всех непустых множеств $P_{\alpha, \beta}^{(\lambda)}$ выполняется неравенство

$$
Q_{\alpha+1} Q_{\beta+1} \leqslant 3 Q_{3} N^{\frac{1}{2}}
$$


Действительно, пусть какое-либо $\Theta$ из $P_{\alpha, \beta}^{(\lambda)}$ имеет параметры а, q и l. Тогда из (7.11) $u$ (7.12) следует, что

$$
Q_{\alpha+1} \leqslant Q_{1} q, Q_{\beta+1} \leqslant Q_{1}|l|
$$

по определению последовательности (7.9). Отсюда и из неравенства (7.5) (имеющего место ввиду (7.12)) следует утверждение замечания.

Определим действительное число $\gamma$ равенством

$$
\gamma=1-\delta_{\mathcal{A}}
$$

и для всякого действительного $x$ положим

$$
\bar{x}=\max \{1,|x|\} .
$$

Лемма 7.2. Пусть найдутся константа $c_{1}=c_{1}\left(\mathcal{A}, \epsilon_{0}\right)>0$ и абсолютная постоянная величина $c_{2}>0$, такие что для любого $\lambda \in\left(-\frac{1}{4}, \frac{1}{4}\right]$, для любых иелых $\alpha, \beta \geqslant 0$, при любом $\epsilon_{0} \in\left(0, \frac{1}{2500}\right)$ выполняется неравенство

$$
\sum_{\Theta \in P_{\alpha, \beta}^{(\lambda)}}\left|S_{N}(\Theta)\right|^{2} \leqslant c_{1} \frac{\left|\Omega_{N}\right|^{2} Q_{\alpha+1}^{2} Q_{\beta+1}}{\bar{Q}_{\alpha}^{3} \bar{Q}_{\beta}^{2}}\left(Q_{\alpha+1} Q_{\beta+1}\right)^{c_{2} \gamma+100 \epsilon_{0}} .
$$

Тогда из оченки

$$
\delta_{\mathcal{A}}>1-\frac{1}{c_{2}}
$$

следуют неравенства (1.4) - (1.6.).

Доказательство. Предположим, что неравенства (7.16) и (7.17) выполнены, и докажем оценки (7.7) и $(7.8)$ для некоторых величин $\epsilon_{0} \in\left(0, \frac{1}{2500}\right)$ и $C=C(\mathcal{A})>0$. Оценим сумму $\Sigma_{N}(Q)$, определенную в $(7.6)$, с помошью введенного в 7.10 множества $P_{\alpha, \beta}^{(\lambda)}$ :

$$
\Sigma_{N}(Q) \leqslant \int_{-\frac{1}{4}}^{\frac{1}{4}} \sum_{\alpha, \beta \geqslant 0} \mathbf{1}_{\left\{Q_{\alpha+1} \geqslant Q \text { или } Q_{\beta+1} \geqslant Q\right\}} \sum_{\Theta \in P_{\alpha, \beta}^{(\lambda)}}\left|S_{N}(\Theta)\right|^{2} d \lambda,
$$

где сумма по $\alpha, \beta$ распространена на целые неотрицательные числа. Подставляя в 7.18 равномерную по $\lambda$ оценку (7.16, получаем:

$$
\Sigma_{N}(Q) \leqslant c_{1} Q_{3}\left|\Omega_{N}\right|^{2} \sum_{\alpha, \beta \geqslant 0} \mathbf{1}_{\left\{Q_{\alpha+1} \geqslant Q \text { или } Q_{\beta+1} \geqslant Q\right\}}\left(Q_{\alpha+1} Q_{\beta+1}\right)^{-1+c_{2} \gamma+100 \epsilon_{0}} .
$$

Определим теперь числа $\epsilon_{0}$ и $C=C(\mathcal{A})$ равенствами

$$
\begin{gathered}
\epsilon_{0}=\min \left\{\frac{1}{200}\left(1-c_{2} \gamma\right), \frac{1}{2600}\right\}, \\
C=C(\mathcal{A})=1-c_{2} \gamma-100 \epsilon_{0} .
\end{gathered}
$$

Тогда, ввиду соотношения (7.17), выполняются неравенства $\epsilon_{0}>0$ и $C>0$. Определим еще две величины: целое число $\xi=\xi(Q) \geqslant 0$ и для произвольных $\alpha_{0}, \beta_{0} \geqslant 0$ - сумму $\Sigma_{N}\left(\alpha_{0}, \beta_{0}\right)$, соответственно, из условий:

$$
Q_{\xi} \leqslant Q<Q_{\xi+1}
$$




$$
\Sigma_{N}\left(\alpha_{0}, \beta_{0}\right)=\sum_{\alpha \geqslant \alpha_{0}} \sum_{\beta \geqslant \beta_{0}}\left(Q_{\alpha+1} Q_{\beta+1}\right)^{-C} .
$$

Тогда, ввиду соотношений (7.19) и (7.20), выполняется оценка

$$
\Sigma_{N}(Q) \ll\left|\Omega_{N}\right|^{2}\left(\Sigma_{N}(0, \xi)+\Sigma_{N}(\xi, 0)\right) .
$$

Заметим, что из неравенства 7.21 следует оценка $Q_{\xi+1} \geqslant \bar{Q}$. В частности, если $\Sigma_{N}\left(\alpha_{0}, \beta_{0}\right)$ - любое из двух слагаемых в скобках правой части неравенства $(7.22)$, то $Q_{\alpha_{0}+1} \geqslant \bar{Q}$ или $Q_{\beta_{0}+1} \geqslant \bar{Q}$. Поэтому из оценки произведения сумм сходящихся геометрических прогрессий

$$
\Sigma_{N}\left(\alpha_{0}, \beta_{0}\right)=\left(\sum_{\alpha \geqslant \alpha_{0}}\left(Q_{\alpha+1}\right)^{-C}\right)\left(\sum_{\beta \geqslant \beta_{0}}\left(Q_{\beta+1}\right)^{-C}\right) \ll\left(Q_{\alpha_{0}+1} Q_{\beta_{0}+1}\right)^{-C}
$$

следуют неравенства

$$
\Sigma_{N}(0, \xi) \ll(\bar{Q})^{-C}, \Sigma_{N}(\xi, 0) \ll(\bar{Q})^{-C}
$$

Подставляя эти оценки в неравенство $(7.22)$, получаем соотношения (7.7) и (7.8). Теперь утверждение настоящей леммы леммы следует из леммы 7.1 и замечания 7.1 . Лемма доказана.

Фиксируем константу

$$
T_{1}=7 Q_{7}
$$

(где $Q_{7}-$ элемент последовательности $(7.9)$ ) и для любого целого $\kappa \in\left[0, T_{1}-1\right]$ положим:

$$
P_{\alpha, \beta}^{(\lambda)}(\kappa)=\left\{\Theta \in P_{\alpha, \beta}^{(\lambda)} \mid l \equiv \kappa \quad\left(\bmod T_{1}\right)\right\} .
$$

Всюду далее $\alpha, \beta \geqslant 0,0 \leqslant \kappa<T_{1}-$ произвольные целые числа, $\lambda \in\left(-\frac{1}{4}, \frac{1}{4}\right]$ произвольное действительное. Через $Z$ всюду далее обозначается произвольное непустое подмножество конечного множества $P_{\alpha, \beta}^{(\lambda)}(\kappa)$. Положим

$$
\sigma_{N, Z}=\sum_{\Theta \in Z}\left|S_{N}(\Theta)\right|
$$

Теорема 7.1. Если найдутся константа $c_{1}=c_{1}\left(\mathcal{A}, \epsilon_{0}\right)>0$ и абсолютная постоянная величина $c_{2}>0$, такие что при любом $\epsilon_{0} \in\left(0, \frac{1}{2500}\right)$ выполняется неравенство

$$
\sigma_{N, Z} \leqslant\left(c_{1}\right)^{\frac{1}{2}} \frac{\left|\Omega_{N}\right||Z|}{\left(\bar{Q}_{\alpha} \bar{Q}_{\beta}|Z|\right)^{\frac{1}{2}}}\left(Q_{\alpha+1} Q_{\beta+1}\right)^{\frac{1}{2}\left(c_{2} \gamma+90 \epsilon_{0}\right)}
$$

то из оценки (7.17) следуют неравенства (1.4) - (1.6).

Доказательство. Достаточно из неравенства (7.26) вывести оценку вида $(7.16)$ с тем же самым значением $c_{2}$, тогда утверждение теоремы будет следовать из леммы 7.2. Вывод неравенства (7.16) легко получается из обобщенной леммы Конягина [10, лемма 13.5]: для всякой функции $f: W \rightarrow \mathbb{R}_{+}$, где $W$ - конечное множество, имеет место неравенство

$$
\sum_{\Theta \in W} f^{2}(\Theta) \ll_{\epsilon}|W|^{\epsilon} \max _{Z \subseteq W}\left(\frac{1}{|Z|}\left(\sum_{\Theta \in Z} f(\Theta)\right)^{2}\right),
$$


где максимум берется по всем непустым подмножествам. Для завершения доказательства остается в (7.27) положить

$$
f(\Theta)=\left|S_{N}(\Theta)\right|, W=P_{\alpha, \beta}^{(\lambda)}(\kappa)
$$

и воспользоваться оценкой 7.26 и неравенством

$$
\sum_{\Theta \in P_{\alpha, \beta}^{(\lambda)}}\left|S_{N}(\Theta)\right|^{2} \leqslant T_{1} \max _{0 \leqslant \kappa \leqslant T_{1}-1} \sum_{\Theta \in P_{\alpha, \beta}^{(\lambda)}(\kappa)}\left|S_{N}(\Theta)\right|^{2} .
$$

Теорема доказана.

Цель всех последующих рассуждений будет состоять в доказательстве неравенства (7.26) при $c_{2}=5$.

\section{8 Общие оценки тригонометрической суммы по ансамблю.}

Всюду в данном параграфе считаем, что числа $M^{(1)}, M^{(2)}, M^{(4)}$, удовлетворяющие условиям теоремы 6.1, уже каким-либо образом выбраны, так что имеет место разложение ансамбля 6.17) (со свойствами 6.18 - 6.23). Именно вид такого разложения отличает данный параграф от аналогичного ему [10, параграф 13] с тем же названием. Ввиду почти дословного соответствия формулировок, доказательство первых двух лемм настоящего параграфа не приводится.

Далее используются обозначения: (6.11), (6.26) и (6.27) из параграфа 6, (7.10) и 7.25) из парагрфа 7, а также обозначения из [10]:

$$
S(x)=3\left(\frac{2 \sin \left(\frac{1}{2} \Pi x\right)}{\Pi x}\right)^{2}, \$(z)=S\left(z_{1}\right) S\left(z_{2}\right)
$$

для $z=\left(z_{1}, z_{2}\right) \in \mathbb{R}^{2}$ при $z \neq(0,0)$, и $\$(0,0)=3$ - по непрерывности.

Лемма 8.1. (см. [10, лемма 13.1]). При некоторых комплексных коэффициентах $\xi(\Theta)$ (где $\Theta \in Z)$, по модулю равных единице, имеет место оценка

$$
\sigma_{N, Z} \ll\left|\Omega^{(1)}\right|^{\frac{1}{2}} \sum_{g_{3} \in \Omega^{(3)}}\left(\sum_{g_{1} \in \mathbb{Z}^{2}} \$\left(\frac{g_{1}}{H}\right)\left|\sum_{\Theta \in Z} \xi(\Theta) \sum_{\widetilde{g} \in \widetilde{\Omega}\left(g_{3}\right)} e\left(g_{1} \widetilde{g} \Theta\right)\right|^{2}\right)^{\frac{1}{2}} .
$$

Отсюда легко получается следующая лемма.

Лемма 8.2. (см. [10, лемма 13.2]). Имеет место оиенка

$$
\sigma_{N, Z} \ll H\left|\Omega^{(1)}\right|^{\frac{1}{2}} \sum_{g_{3} \in \Omega^{(3)}}\left(\sum_{\substack{g^{(1)}, g^{(2)} \in \tilde{\Omega}_{(g)} \\ \Theta^{(1)}, \Theta^{(2)} \in Z}} 1_{\left\{\left\|\widetilde{g}^{(1)} \Theta^{(1)}-\widetilde{g}^{(2)} \Theta^{(2)}\right\|_{1,2} \leqslant \frac{1}{2 H}\right\}}\right)^{\frac{1}{2}},
$$

где $\|z\|_{1,2}=\max \left\{\left\|z_{1}\right\|,\left\|z_{2}\right\|\right\}$ для $z=\left(z_{1}, z_{2}\right)^{t} \in \mathbb{R}^{2}$. 
Напомним, что всегда $Z \subseteq P_{\alpha, \beta}^{(\lambda)}(\kappa)$ для соответствующих значений параметров. Числа $\Theta_{1}, \Theta_{2} \in P_{\alpha, \beta}^{(\lambda)}(\kappa)$ будем далее записывать следующим образом:

$$
\Theta^{(1)}=\frac{a^{(1)}}{q^{(1)}}+\frac{l^{(1)}}{2 N}+\frac{\lambda}{N}, \quad \Theta^{(2)}=\frac{a^{(2)}}{q^{(2)}}+\frac{l^{(2)}}{2 N}+\frac{\lambda}{N} .
$$

Положим:

$$
\left.\mathfrak{N}\left(g_{3}\right)=\left\{\left(g^{(1)}, g^{(2)}, \Theta^{(1)}, \Theta^{(2)}\right) \in\left(\widetilde{\Omega}\left(g_{3}\right)\right)^{2} \times Z^{2} \mid 8.3\right) \text { и 8.4 выполнены для } i=1,2\right\},
$$

где (в обозначениях из 6.27)):

$$
\begin{gathered}
\left\|\frac{x_{i} a^{(1)}}{q^{(1)}}-\frac{y_{i} a^{(2)}}{q^{(2)}}\right\| \leqslant \min \left\{\begin{array}{l}
\frac{1}{N} 150 A^{3} x_{i}+\left\|\frac{1}{2 N}\left(x_{i} l^{(1)}-y_{i} l^{(2)}\right)\right\|+\left\|\frac{\lambda}{N}\left(x_{i}-y_{i}\right)\right\|, \\
\frac{1}{N}(9 A)^{5} x_{i} Q_{\beta+1}, \\
\frac{1}{M^{(1)}} 74 A^{2} Q_{\beta+1},
\end{array}\right. \\
\qquad x_{i} l^{(1)}-y_{i} l^{(2)} \mid \leqslant(9 A)^{5} x_{i}+2 N\left\|x_{i} \frac{a^{(1)}}{q^{(1)}}-y_{i} \frac{a^{(2)}}{q^{(2)}}\right\| .
\end{gathered}
$$

Лемма 8.3. (см. [10, лемма 13.3]). Если выполнено неравенство

$$
M^{(1)} \geqslant 150 A^{2} Q_{\beta+1}
$$

то имеет место оченка

$$
\sigma_{N, Z} \ll H\left|\Omega^{(1)}\right|^{\frac{1}{2}} \sum_{g_{3} \in \Omega^{(3)}}\left|\mathfrak{N}\left(g_{3}\right)\right|^{\frac{1}{2}}
$$

Доказательство. Будем выводить оценки 8.3 и 8.4 из неравенства в фигурных скобках в 8.1): тогда утверждение настоящей леммы будет следовать из предыдущей.

Применение верхней оценки в 7.12 или неравенства $|\lambda| \leqslant \frac{1}{4}$ дает, соответственно, неравенства:

$$
\begin{gathered}
\left|\frac{x_{i} l^{(1)}}{2 N}-\frac{y_{i} l^{(2)}}{2 N}\right| \leqslant \frac{Q_{\beta+1}}{N} \max \left\{x_{i}, y_{i}\right\}, \\
\left|\frac{x_{i} \lambda}{N}-\frac{y_{i} \lambda}{N}\right| \leqslant \frac{2|\lambda|}{N} \max \left\{x_{i}, y_{i}\right\} \leqslant \frac{1}{2 N} \max \left\{x_{i}, y_{i}\right\} .
\end{gathered}
$$

Ввиду обозначений, введенных равенствами (6.25) и 6.26), имеет место включение $\Omega\left(g_{3}\right) \subseteq \Omega$. Следовательно, к максимуму из двух последних неравенств можно применить любую из двух верхних оценок: ту, которая в 6.20, или 6.29). Их применение дает:

$$
\begin{gathered}
\left|\frac{x_{i} l^{(1)}}{2 N}-\frac{y_{i} l^{(2)}}{2 N}\right| \leqslant \min \left\{\frac{73 A^{2} Q_{\beta+1}}{M^{(1)}}, \frac{1}{N} 22000 A^{5} Q_{\beta+1} x_{i}\right\}, \\
\left\|\frac{x_{i} \lambda}{N}-\frac{y_{i} \lambda}{N}\right\| \leqslant \min \left\{\frac{73 A^{2}}{2 M^{(1)}}, \frac{1}{N} 11000 A^{5} x_{i}\right\} .
\end{gathered}
$$

Из неравенства 8.5 следует, что первый элемент минимума в 8.7 меньше, чем $\frac{1}{2}$, поэтому

$$
\left\|\frac{x_{i} l^{(1)}}{2 N}-\frac{y_{i} l^{(2)}}{2 N}\right\|=\left|\frac{x_{i} l^{(1)}}{2 N}-\frac{y_{i} l^{(2)}}{2 N}\right| .
$$


Применяя дважды неравенство треугольника, получаем:

$$
\begin{aligned}
& \left\|\frac{x_{i} a^{(1)}}{q^{(1)}}-\frac{y_{i} a^{(2)}}{q^{(2)}}\right\| \leqslant\left\|\frac{x_{i} l^{(1)}}{2 N}-\frac{y_{i} l^{(2)}}{2 N}\right\|+\left\|\frac{x_{i} \lambda}{N}-\frac{y_{i} \lambda}{N}\right\|+\left\|x_{i} \Theta^{(1)}-y_{i} \Theta^{(2)}\right\|, \\
& \left\|\frac{x_{i} l^{(1)}}{2 N}-\frac{y_{i} l^{(2)}}{2 N}\right\| \leqslant\left\|\frac{x_{i} a^{(1)}}{q^{(1)}}-\frac{y_{i} a^{(2)}}{q^{(2)}}\right\|+\left\|\frac{x_{i} \lambda}{N}-\frac{y_{i} \lambda}{N}\right\|+\left\|x_{i} \Theta^{(1)}-y_{i} \Theta^{(2)}\right\| .
\end{aligned}
$$

Заметим, что из (8.10) сразу получается верхняя строчка из минимума в (8.3), если третье слагаемое в правой части 8.10 оценить, как в неравенстве 8.1):

$$
\left\|x_{i} \Theta^{(1)}-y_{i} \Theta^{(2)}\right\| \leqslant \frac{1}{2 H} \leqslant \frac{1}{N} 150 A^{3} x_{i}
$$

ввиду оценки 6.30). Если теперь остальные слагаемые из правой части 8.10 оценить вторыми элементами минимумов в неравенствах (8.7) и (8.8), то получим вторую строку минимума в (8.3). Далее, третья строчка минимума в (8.3) получается применением в неравенстве (8.10) первых элементов минимумов из неравенств (8.7) и (8.8). Теперь неравенство (8.3) полностью доказано.

Аналогично, оценка правой части (8.11) производится на основании второго элемента минимума в (8.8), а также неравенства (8.12). К результату применяется равенство (8.9), приводящее к оценке (8.4). Лемма доказана.

Обозначим:

$\mathfrak{M}\left(g_{3}\right)=\left\{\left(\widetilde{g}^{(1)}, \widetilde{g}^{(2)}, \Theta^{(1)}, \Theta^{(2)}\right) \in\left(\widetilde{\Omega}\left(g_{3}\right)\right)^{2} \times Z^{2}|8.13| 8.14\right.$ и выполнены для $\left.i=1,2\right\}$, где (в обозначениях 6.27 и 8.2 )

$$
\begin{gathered}
\left\|\frac{x_{i} a^{(1)}}{q^{(1)}}-\frac{y_{i} a^{(2)}}{q^{(2)}}\right\|=0, \\
\left|x_{i} l^{(1)}-y_{i} l^{(2)}\right| \leqslant(9 A)^{5} x_{i} .
\end{gathered}
$$

Далее через q обозначается наименьшее общее кратное чисел $q^{(1)}$ и $q^{(2)}$.

Замечание 8.1. В работе [1, пn. 6.2.1] (см. также [10, доказательство леммы 14.1]) было показано, что условие (8.13) равносильно следующему:

$$
q^{(1)}=q^{(2)}=\mathbf{q}, x_{i} a^{(1)} \equiv y_{i} a^{(2)} \quad(\bmod \mathbf{q}), i=1,2 .
$$

Отсюда следует, что для элементов множества $\mathfrak{M}\left(g_{3}\right)$ из равенства $x_{1}=y_{1}$ следуют соотношения: $a^{(1)}=a^{(2)}-$ в виду сравнения в 8.15) nри $i=1$, а равенство $l^{(1)}=l^{(2)}$ - в виду неравенства (8.14) при $i=1$ и сравнения в (7.24).

Лемма 8.4. (см. [10, лемма 13.4]). Пусть число $M^{(1)}$ определено равенством

$$
M^{(1)}=150 A^{2} Q_{\alpha+1}^{2} Q_{\beta+1} .
$$

Тогда при любом $g_{3} \in \Omega^{(3)}$ имеет место включение

$$
\mathfrak{N}\left(g_{3}\right) \subseteq \mathfrak{M}\left(g_{3}\right)
$$


Доказательство. Покажем, что соотношения (8.13) и (8.14) в условиях леммы следуют из неравенств (8.3) и 8.4). Действительно, в виду (8.16), третья строка минимума в правой части неравенства (8.3) меньше, чем $\frac{1}{q_{1} q_{2}}-$ следовательно, оцениваемая величина равна нулю. Отсюда следует равенство (8.13), подстановка которого в (8.4) дает неравенство 8.14). Лемма доказана.

Введем следующие обозначения (для переменных из (6.27)):

$$
\begin{gathered}
\mathcal{Y}=x_{1} y_{2}-x_{2} y_{1}, \\
Y=Y\left(M^{(1)}\right)=\frac{75 A^{2} N}{M^{(1)}} .
\end{gathered}
$$

Лемма 8.5. (см. [1, доказательство предложения 6.11]). Пусть число $M^{(1)}$ определено равенством

$$
M^{(1)}=120 A^{2}\left(N Q_{\alpha+1} Q_{\beta+1}\right)^{\frac{1}{2}} .
$$

Тогда при любом $g_{3} \in \Omega^{(3)}$ для элементов множества $\mathfrak{N}\left(g_{3}\right)$ имеют место соотношения

$$
\begin{gathered}
0<x_{i}<Y, \quad 0<y_{i}<Y, \quad i=1,2, \\
\mathcal{Y}<Y^{2} \\
\frac{2 Y^{2} Q_{\beta+1}}{N}<\frac{1}{Q_{\alpha+1}}, \\
\mathcal{Y} \equiv 0 \quad(\bmod \mathbf{q}) .
\end{gathered}
$$

Доказательство. Неравенства (8.21) следуют из верхней оценки в (6.20), откуда следует неравенство 8.22). Далее, соотношение 8.23) проверяется непосредственным применением равенств (8.19) и 8.20). Наконец, поскольку

$$
\left\|\mathcal{Y} \frac{a^{(1)}}{q^{(1)}}\right\| \leqslant y_{2}\left\|\frac{x_{1} a^{(1)}}{q^{(1)}}-\frac{y_{1} a^{(2)}}{q^{(2)}}\right\|+y_{1}\left\|\frac{x_{2} a^{(1)}}{q^{(1)}}-\frac{y_{2} a^{(2)}}{q^{(2)}}\right\|
$$

то, применяя здесь нижнюю строку минимума в (8.3) и оценки (8.21), получаем:

$$
\left\|\mathcal{Y} \frac{a^{(1)}}{q^{(1)}}\right\|<2 Y \frac{74 A^{2} Q_{\beta+1}}{M^{(1)}}<\frac{2 Y^{2} Q_{\beta+1}}{N}<\frac{1}{Q_{\alpha+1}}<\frac{1}{q^{(1)}}
$$

ввиду доказанной оценки 8.23 . Следовательно, $\left\|\mathcal{Y} \frac{a^{(1)}}{q^{(1)}}\right\|=0$, то есть $\mathcal{Y}$ делится на $q^{(1)}$. Аналогично, $\mathcal{Y}$ делится на $q^{(2)}$, откуда следует сравнение 8.24 . Лемма доказана.

Лемма 8.6. Если число $M^{(1)}$ определено равенством (8.20), по при любом $g_{3} \in \Omega^{(3)}$ имеет место включение (8.17).

Доказательство. Как и в доказательстве леммы 8.4, достаточно лишь вывести равенство 8.13) из соотношений 8.3, 8.4 и условий настоящей леммы.

Пусть для начала $\mathcal{Y}$ не равно нулю (случай 1, см. [1, пп. 6.2.1]). Тогда, ввиду соотношений (8.22 и 8.24), имеет место цепочка неравенств:

$$
\mathbf{q} \leqslant \min \left\{Q_{\alpha+1}^{2},|\mathcal{Y}|\right\} \leqslant \min \left\{Q_{\alpha+1}^{2}, Y^{2}\right\} \leqslant Q_{\alpha+1} Y .
$$


Отсюда и из (8.23) следует, что

$$
\frac{Y Q_{\beta+1}}{N} \leqslant \frac{Y^{2} Q_{\alpha+1} Q_{\beta+1}}{\mathbf{q} N}<\frac{1}{\mathbf{q}} .
$$

Поэтому третья строчка минимума в 8.3 меньше, чем $\frac{1}{\mathbf{q}}$. Следовательно, левая часть неравенства (8.3) равна нулю, так что имеет место неравенство (8.13).

Пусть теперь $\mathcal{Y}$ равно нулю (случай 2, см. [10, доказательство леммы 14.6]). Ввиду взаимной простоты чисел $x_{1}$ и $x_{2}$, а также $y_{1}$ и $y_{2}$, это означает, что

$$
x_{1}=y_{1}, \quad x_{2}=y_{2} .
$$

Поэтому любая из первых двух строчек минимума в 8.3 может быть представлена в виде $F\left(x_{i}\right)$ для $i=1,2$, где $F$ - некоторая однородная линейная функция. Следовательно, если положить $\xi=\left|\frac{a^{(1)}}{q^{(1)}}-\frac{a^{(2)}}{q^{(2)}}\right|$, то оценка 8.3 запишется в виде

$$
\left\|x_{i} \xi\right\| \leqslant F\left(x_{i}\right)
$$

Другими словами, если $n_{1}$ и $n_{2}$ - ближайшие целые числа к величинам $x_{1} \xi$ и $x_{2} \xi$, соответственно, то найдутся действительные числа $\Theta_{1}, \Theta_{2}$, такие что $\left|\Theta_{1}\right|,\left|\Theta_{2}\right|<\frac{1}{2}$ и

$$
n_{1}=x_{1} \xi+\Theta_{1} F\left(x_{1}\right), \quad n_{2}=x_{2} \xi+\Theta_{2} F\left(x_{2}\right) .
$$

В частности, если $F\left(x_{i}\right)$ - вторая строка минимума в 8.3), то из 8.21) и 8.30) следует, что

$$
\left|x_{1} n_{2}-x_{2} n_{1}\right|=x_{1} x_{2}\left|\Theta_{1}-\Theta_{2}\right| \frac{(9 A)^{5} Q_{\beta+1}}{N} \leqslant \frac{Y^{2}(9 A)^{5} Q_{\beta+1}}{N} \leqslant \frac{(9 A)^{5}}{Q_{\alpha+1}}<1
$$

ввиду 8.23) (и поскольку $\left.(9 A)^{5}<Q_{1} \leqslant Q_{\alpha+1}\right)$. Следовательно, $x_{1} n_{2}=x_{2} n_{1}$. Но числа $x_{1}$ и $x_{2}$ взаимно просты, поэтому найдется целое число $k$, такое что $n_{2}=k x_{2}$. Отсюда, возвращаясь к случаю произвольного из двух вариантов для $F\left(x_{2}\right)$ во втором из равенств в 8.30) и сокращая на $x_{2}$, получаем:

$$
\|\xi\| \leqslant|\xi-k| \leqslant \frac{F\left(x_{2}\right)}{x_{2}}
$$

В частности, при $Q_{\alpha+1}<\frac{1}{Q_{4}} N^{\frac{1}{2}}$ (случай 2.1), если $F\left(x_{i}\right)$ - вторая строка минимума в (8.3), то из неравенств (8.31) и 7.13 получаем:

$$
\|\xi\| \leqslant \frac{(9 A)^{5} Q_{\beta+1}}{N} \leqslant \frac{3(9 A)^{5} Q_{3}}{Q_{\alpha+1} N^{\frac{1}{2}}}<\frac{Q_{4}}{Q_{\alpha+1} N^{\frac{1}{2}}}<\frac{1}{Q_{\alpha+1}^{2}} \leqslant \frac{1}{\mathbf{q}},
$$

откуда $\xi=0$.

Наоборот, пусть теперь $Q_{\alpha+1} \geqslant \frac{1}{Q_{4}} N^{\frac{1}{2}}$ (случай 2.2). Тогда, ввиду 7.12 и 7.13, при $l=l^{(1)}, l^{(2)}$ выполнено неравенство

$$
|l| \leqslant Q_{\beta+1} \leqslant \frac{3 Q_{3} N^{\frac{1}{2}}}{Q_{\alpha+1}} \leqslant 3 Q_{7}
$$

Поэтому, в обозначениях (7.23), имеет место оценка

$$
\left|l^{(1)}-l^{(2)}\right| \leqslant 6 Q_{7}<7 Q_{7}=T_{1} .
$$


Однако сравнение в 7.24 показывает, что $l^{(1)} \equiv l^{(2)}\left(\bmod T_{1}\right)$. Вместе с неравенством 8.34 это означает, что $l^{(1)}=l^{(2)}$. Возвращаясь теперь к неравенству 8.31 , заменим в нем $F\left(x_{2}\right)$ на первую строку минимума в 8.3 , равную, ввиду 8.29 , числу $\frac{1}{N} 150 A^{3} x_{2}$. Тогда получим:

$$
\|\xi\| \leqslant \frac{150 A^{3}}{N}<\frac{1}{q^{(1)} q^{(2)}} \leqslant \frac{1}{\mathbf{q}}
$$

ввиду неравенства в 7.3 , откуда снова получаем равенство $\xi=0$. Таким образом, $\frac{a^{(1)}}{q^{(1)}}=\frac{a^{(2)}}{q^{(2)}}$. Отсюда и из 8.29 следует равенство 8.13 . Лемма доказана.

Теорема 8.1. Пусть число $M^{(1)}$ определено любым из равенств (8.16) или (8.20). Тогда в условиях теоремы 6.1 имеет место оценка

$$
\sigma_{N, Z} \ll\left(M^{(1)}\right)^{1+\epsilon_{0}}\left|\Omega^{(1)}\right|^{\frac{1}{2}} \sum_{g_{3} \in \Omega^{(3)}}\left|\mathfrak{M}\left(g_{3}\right)\right|^{\frac{1}{2}} .
$$

Доказательство. Из определения числа $M^{(1)}$ следует, что неравенство 8.5 выполнено по крайней мере для всех достаточно больших значений $N$. Следовательно, условия леммы 8.3 выполнены. Значит, имеет место неравенство 8.6). Отсюда и из включения 8.17), доказанного в леммах 8.4 и 8.6. следует неравенство (8.35) (если учесть определение числа $H$ в (6.11)). Теорема доказана.

Тем самым задача об оценке тригонометрической суммы сведена к определению мощности множества $\mathfrak{M}\left(g_{3}\right)$.

\section{9 Исследование и применение множества $\mathfrak{M}\left(g_{3}\right)$}

В этом параграфе будет получено значение мощности множества $\mathfrak{M}\left(g_{3}\right)$, что позволит вывести полезные следствия из соотношения (8.35). Всюду далее число $M^{(1)}$ определено одним из двух вариантов - как в теореме 8.1

Напомним обозначения (6.12) и (7.9) и при $\beta \geqslant 0$ положим:

$$
\begin{gathered}
M_{\beta}^{(2)}=\frac{1}{Q_{3}}\left(M^{(1)}\right)^{-2 \epsilon_{0}}\left(Q_{\beta}\right)^{\frac{1}{2}-2 \epsilon_{0}}, \\
M^{(2)}=\left\{\begin{array}{l}
M_{\beta}^{(2)}, \text { если } M_{\beta}^{(2)} \geqslant Q_{1}, \\
1,- \text { в противном случае. }
\end{array}\right.
\end{gathered}
$$

В следующих ниже леммах $9.1-9.3$ свойства (6.16) - 6.23) продолжаем считать выполненными.

Лемма 9.1. Пусть число $M^{(2)}$ определено соотношением (9.2). Тогда при любом $g_{3} \in \Omega^{(3)}$ для каждого элемента множества $\mathfrak{M}\left(g_{3}\right)$ выполняется равенство

$$
g_{2}^{(1)}=g_{2}^{(2)}
$$

Доказательство. Если $M^{(2)}=1$, то $\Omega^{(2)}=\{E\}$, так что утверждение леммы было бы очевидно. В частности, если $Q_{\beta}=0$, то снова $M^{(2)}=1$, и лемма была бы доказана.

Пусть теперь утверждение леммы не имеет места. Тогда

$$
M^{(2)}=M_{\beta}^{(2)} \geqslant Q_{1}>1,\left|l^{(1)}\right|,\left|l^{(2)}\right| \geqslant Q_{\beta}>0,
$$


и найдется четверка

$$
\left(\widetilde{g}^{(1)}, \widetilde{g}^{(2)}, \Theta^{(1)}, \Theta^{(2)}\right) \in \mathfrak{M}\left(g_{3}\right),
$$

для которой $g_{2}^{(1)} \neq g_{2}^{(2)}$. Последнее неравенство, ввиду взаимной однозначности отображения (6.1), имеет особую интерпретацию в обозначениях (6.27). Именно, не только выполнено соотношение $\frac{x_{1}}{x_{2}} \neq \frac{y_{1}}{y_{2}}$. Более того, если последовательность $D$ (возможно, пустая) - максимальная общая часть последовательностей неполных частных для цепных дробей

$$
\frac{x_{1}}{x_{2}}=[D, T], \quad \frac{y_{1}}{y_{2}}=[D, W],
$$

то последовательность $D$ не длиннее, чем те последовательности, которым соответствуют матрицы $g_{2}^{(1)}$ и $g_{2}^{(2)}$ в смысле отображения (??). Следовательно, из 6.21 имеем оценKy:

$$
\langle D\rangle \leqslant \max _{g_{2} \in \Omega^{(2)}}\left\|g_{2}\right\| \leqslant 73 A^{2} M^{(2)}\left(M^{(1)} M^{(2)}\right)^{2 \epsilon_{0}} .
$$

Кроме того, длины цепных дробей (9.6) совпадают по построению ансамбля. Следовательно, выполнены условия леммы 5.1, применяя которую, получаем:

$$
\left|\frac{x_{1}}{x_{2}}-\frac{y_{1}}{y_{2}}\right| \geqslant \frac{1}{(2 A)^{4}\langle D\rangle^{2}} \geqslant \frac{1}{(2 A)^{4}\left(73 A^{2} M^{(2)}\left(M^{(1)} M^{(2)}\right)^{2 \epsilon_{0}}\right)^{2}} \geqslant \frac{(5 A)^{-8}\left(M^{(1)}\right)^{-4 \epsilon_{0}}}{\left(M^{(2)}\right)^{2+4 \epsilon_{0}}}
$$

ввиду 9.7. Подставляя сюда выражение для $M^{(2)}=M_{\beta}^{(2)}$ из 9.1 и применяя оценку $Q_{3} \geqslant(7 A)^{7}$, получаем:

$$
\left|\frac{x_{1}}{x_{2}}-\frac{y_{1}}{y_{2}}\right| \geqslant \frac{(7 A)^{7\left(2+4 \epsilon_{0}\right)}}{(5 A)^{8} Q_{\beta}}>\frac{2(9 A)^{5}}{Q_{\beta}} .
$$

С другой стороны, применяя при $i=1,2$ неравенство (8.14), получаем:

$$
\left|\frac{x_{1}}{x_{2}}-\frac{y_{1}}{y_{2}}\right| \leqslant \frac{y_{1}\left|x_{2} l^{(1)}-y_{2} l^{(2)}\right|+y_{2}\left|x_{1} l^{(1)}-y_{1} l^{(2)}\right|}{x_{2} y_{2} l^{(1)}} \leqslant \frac{(9 A)^{5}}{l^{(1)}}\left(\frac{y_{1}}{y_{2}}+\frac{x_{1}}{x_{2}}\right) \leqslant \frac{2(9 A)^{5}}{Q_{\beta}}
$$

(напомним, что $|l|^{(1)}>0$ ввиду $(9.4)$ ), что противоречит неравенству $(9.9)$. К этому противоречию привело предположение о том, что $g_{2}^{(1)} \neq g_{2}^{(2)}$. Следовательно, $g_{2}^{(1)}=g_{2}^{(2)}$. Лемма доказана.

Для $\alpha \geqslant 0$ положим:

$$
\begin{gathered}
M_{\alpha}^{(4)}=\frac{1}{Q_{3}}\left(Q_{\alpha}\right)^{\frac{1}{2}}, \\
M^{(4)}=\left\{\begin{array}{l}
M_{\alpha}^{(4)}, \text { если } M_{\alpha}^{(4)} \geqslant Q_{1}, \\
1,- \text { в противном случае. }
\end{array}\right.
\end{gathered}
$$

Всюду далее числа $M^{(2)}$ и $M^{(4)}$ определены соотношениями 9.2 и 9.11 , соответственно.

Лемма 9.2. При любом $g_{3} \in \Omega^{(3)}$ для каждого элемента множества $\mathfrak{M}\left(g_{3}\right)$ выполняются равенства (9.3) и

$$
\widetilde{g}_{4}^{(1)}=\widetilde{g}_{4}^{(2)}
$$


Доказательство. Если $M^{(4)}=1$, то $\Omega^{(4)}=\{E\}$, так что равенство 9.12 было бы очевидно. В частности, если $Q_{\alpha}=1$, то снова $M^{(4)}=1$, и лемма была бы доказана.

Пусть теперь утверждение леммы не имеет места. Тогда $M^{(4)}=M_{\alpha}^{(4)} \geqslant Q_{1}>1$, $\mathbf{q} \geqslant Q_{\alpha}>1$ и, следовательно, $a^{(1)}>0$.

Равенство 9.3), согласно которому $g_{2}^{(1)}=g_{2}^{(2)}=g_{2}$, уже доказано в предыдущей лемме. Следовательно, сравнения в 8.15 могут быть записаны в виде

$$
\left(g_{2} g_{3} a^{(1)} \widetilde{g}_{4}^{(1)} \equiv g_{2} g_{3} a^{(2)} \widetilde{g}_{4}^{(2)}\right)_{1,2} \quad(\bmod \mathbf{q})
$$

где индексы " 1,2 " внизу означают, как всегда, выполнение сравнения по обеим координатам. Отсюда, в виду равенства $\operatorname{det} g_{2} g_{3}=1$, получаем:

$$
\left(a^{(1)} \widetilde{g}_{4}^{(1)} \equiv a^{(2)} \widetilde{g}_{4}^{(2)}\right)_{1,2} \quad(\bmod \mathbf{q})
$$

Положим $\widetilde{g}_{4}^{(1)}=\left(u_{1}, u_{2}\right)^{t}, \quad \widetilde{g}_{4}^{(2)}=\left(v_{1}, v_{2}\right)^{t}$, тогда сравнения 9.13 перепишутся в виде

$$
\begin{aligned}
& a^{(1)} u_{1} \equiv a^{(2)} v_{1} \quad(\bmod \mathbf{q}), \\
& a^{(1)} u_{2} \equiv a^{(2)} v_{2} \quad(\bmod \mathbf{q}) .
\end{aligned}
$$

Отсюда следует цепочка сравнений:

$$
\left(a^{(1)} u_{2}\right) v_{1} \equiv a^{(2)} v_{2} v_{1}=\left(a^{(2)} v_{1}\right) v_{2} \equiv\left(a^{(1)} u_{1}\right) v_{2} \quad(\bmod \mathbf{q})
$$

Но числа $a^{(1)}$ и $\mathbf{q}$ взаимно просты как числитель и знаменатель цепной дроби (и при этом $a^{(1)}>0$, как уже было сказано выше). Следовательно, сокращая начало и конец цепочки 9.14 на $a^{(1)}$, получаем:

$$
u_{2} v_{1}-u_{1} v_{2} \equiv 0 \quad(\bmod \mathbf{q})
$$

С другой стороны,

$$
\left|u_{2} v_{1}-u_{1} v_{2}\right| \leqslant u_{2} v_{2} \leqslant\left(\max _{g \in \Omega^{(4)}}\|g\|\right)^{2}
$$

Применяя к окончанию соотношения (9.16) верхнюю оценку из неравенства (6.22), получаем:

$$
\left|u_{2} v_{1}-u_{1} v_{2}\right| \leqslant\left(73 A^{2} M^{(4)}\right)^{2} \leqslant(9 A)^{4}\left(M^{(4)}\right)^{2} .
$$

Подставляя в последнее неравенство значение $M^{(4)}=M_{\alpha}^{(4)}$ из 9.10 , получаем:

$$
\left|u_{2} v_{1}-u_{1} v_{2}\right|<Q_{\alpha} \leqslant \mathbf{q} .
$$

ввиду нижней оценки из неравенства 7.11. Соотношения 9.15) и 9.17 показывают, что

$$
u_{2} v_{1}=u_{1} v_{2}
$$

Отсюда, вследствие несократимости дробей $\frac{u_{1}}{u_{2}}, \frac{v_{1}}{v_{2}}$, получаем: $u_{1}=v_{1}, u_{2}=v_{2}$, или $\widetilde{g}_{4}^{(1)}=\widetilde{g}_{4}^{(2)}$, так что равенство 9.12 доказано. Лемма доказана.

Лемма 9.3. При любом $g_{3} \in \Omega^{(3)}$ имеет место равенство

$$
\left|\mathfrak{M}\left(g_{3}\right)\right|=\left|\Omega^{(2)}\right|\left|\Omega^{(4)}\right||Z| .
$$


Доказательство. Пересчитаем четверки элементов (9.5), пользуясь предыдущей леммой. Для этого выберем и фиксируем матрицы $g_{2}^{(1)}=g_{2}^{(2)}$ и $g_{4}^{(1)}=g_{4}^{(2)}$, определяющие элементы $\widetilde{g}^{(1)}=\widetilde{g}^{(2)}$, одним из $\left|\Omega^{(2)}\right|\left|\Omega^{(4)}\right|$ способов - это первые два множителя в (9.18). Выберем также число $\Theta^{(2)} \in Z$, как в 8.2$),|Z|$ способами - это третий и последний множитель в (9.18). Заметим теперь, что из равенства $\widetilde{g}^{(1)}=\widetilde{g}^{(2)}$ (вытекающего из предыдущей леммы) следует, что $x_{1}=y_{1}$. Поэтому, согласно замечанию 8.1. выполняются равенства $a^{(1)}=a^{(2)}$ и $l^{(1)}=l^{(2)}$. Подстановка последних в 8.2 приводит к соотношению $\Theta^{(1)}=\Theta^{(2)}$, завершающему вывод формулы 9.18$)$. Лемма доказана.

Далее выполнение условий теоремы 6.1 заранее не предполагается. Напомним обозначения (7.14) и 7.15 .

Теорема 9.1. Для всякого числа $M^{(1)}$, определенного любым из равенств (8.16) или (8.20), из неравенства

$$
M^{(1)} M_{\beta}^{(2)} M_{\alpha}^{(4)} \leqslant N
$$

следует оценка

$$
\sigma_{N, Z} \ll \frac{\left|\Omega_{N}\right||Z|}{\left(\bar{Q}_{\alpha} \bar{Q}_{\beta}|Z|\right)^{\frac{1}{2}}}\left(M^{(1)} Q_{\alpha+1}^{\frac{1}{2}} Q_{\beta+1}^{\frac{1}{2}}\right)^{\gamma+8 \epsilon_{0}}
$$

Доказательство. Ввиду неравенства (9.19), выполнено условие 6.13) теоремы 6.1, согласно которой найдется разложение ансамбля 6.17) со свойствами (6.18) - 6.23). Следовательно, для оценки величины $\sigma_{N, Z}$ имеем право применять как теорему 8.1. так и лемму 9.3 , утверждения которых приводят к неравенству:

$$
\sigma_{N, Z} \ll\left(M^{(1)}\right)^{1+2 \epsilon_{0}}\left|\Omega^{(1)}\right|^{\frac{1}{2}} \sum_{g_{3} \in \Omega^{(3)}}\left|\Omega^{(2)}\right|^{\frac{1}{2}}\left|\Omega^{(4)}\right|^{\frac{1}{2}}|Z|^{\frac{1}{2}} .
$$

Отсюда, применяя соотношения 6.17 - 6.18), получаем:

$$
\sigma_{N, Z} \ll \frac{\left(M^{(1)}\right)^{1+2 \epsilon_{0}}\left|\Omega_{N}\right||Z|^{\frac{1}{2}}}{\left|\Omega^{(1)} \Omega^{(2)}\right|^{\frac{1}{2}}\left|\Omega^{(4)}\right|^{\frac{1}{2}}} \ll \frac{\left(M^{(1)}\right)^{1+2 \epsilon_{0}}\left|\Omega_{N}\right||Z|^{\frac{1}{2}}}{\left(M^{(1)} M^{(2)} M^{(4)}\right)^{\delta_{\mathcal{A}}-\epsilon_{0}}} .
$$

Подставляя в неравенство 9.21 определения величин $M^{(2)}$ и $M^{(4)}$, получаем оценку (9.20). Теорема доказана.

\section{0 Доказательство теорем 2.1 и 2.2}

Согласно теореме 7.1, для доказательства обеих теорем из заголовка параграфа достаточно доказать неравенство $(7.26)$ при $c_{2}=5$. Будем выводить оценку $(7.26)$ из неравенства 9.20) теоремы 9.1 .

Для начала определим число $M^{(1)}$ равенством 8.20, тогда проверка неравенства 9.19) легко получается применением замечания 7.2. Следовательно, выполнено условие теоремы 9.1. применение которой приводит к оценке

$$
\sigma_{N, Z} \ll \frac{\left|\Omega_{N}\right||Z|}{\left(\bar{Q}_{\alpha} \bar{Q}_{\beta}|Z|\right)^{\frac{1}{2}}}\left(N^{\frac{1}{2}} Q_{\alpha+1} Q_{\beta+1}\right)^{\gamma} N^{12 \epsilon_{0}} .
$$

Пусть (случай 1) выполнено неравенство

$$
N<\left(Q_{\alpha+1}\right)^{\frac{5}{2}}\left(Q_{\beta+1}\right)^{\frac{3}{2}} .
$$


Тогда, ввиду 10.2), имеет место оценка

$$
N^{\frac{1}{2}} Q_{\alpha+1} Q_{\beta+1}<\left(Q_{\alpha+1}\right)^{\frac{9}{4}}\left(Q_{\beta+1}\right)^{\frac{7}{4}}<\left(Q_{\alpha+1}\right)^{\frac{5}{2}}\left(Q_{\beta+1}\right)^{\frac{5}{2}},
$$

подстановка которой в неравенство (10.1) приводит к нужной оценке $(7.26)$ с $c_{2}=5$.

Если же неравенство (10.2 не выполнено (случай 2), то, следовательно, имеет место противоположное неравенство

$$
\left(Q_{\alpha+1}\right)^{\frac{5}{2}}\left(Q_{\beta+1}\right)^{\frac{3}{2}} \leqslant N
$$

В этом случае для числа $M^{(1)}$, определенного равенством 8.16 , выполнено неравенство (9.19). Следовательно, имеем право применить теорему 9.1, которая при подстановке такого значения $M^{(1)}$ приводит к оценке 7.26 с $c_{2}=5$. Доказательство теорем закончено.

\section{Список литературы}

[1] J. Bourgain, A. Kontorovich. On Zaremba's conjecture. Annals Math., 2013.

[2] D. Hensley. The Hausdorff dimensions of some continued fraction cantor sets, J. Number Theory 33:2 (1989), 182-198

[3] O. Jenkinson. On the density of Hausdorff dimensions of bounded type continued fraction sets: the Texan conjecture. Stochastics and Dynamics, 4 (2004), 63-76.

[4] S.K. Zaremba. La méthode des "bons treillis"pour le calcul des intégerales multiples. Applications of number theory to numerical analysis (Montreal, Canada, 1971), pages 39-119, Academic Press, New York, 1972.

[5] R. Graham, D. Knuth, O. Patashnik. Concrete Mathematics: A Foundation for Computer Science, Addison-Wesley, Reading, MA, 1994.

[6] N.G. Moshchevitin. On some open problems in diophantine approximation, preprint available at arXiv:1202.4539v4

[7] Н. М. КороБов. Теоретико-числовые методы в приближенном анализе. Физматгиз, M., 1963.

[8] D.A. Frolenkov, I.D. Kan. A reinforsment of the Bourgain-Kontorovich's theorem by elementary methods. Preprint available at arXiv: abs/1207.4546.

[9] D.A. Frolenkov, I.D. Kan. A reinforsment of the Bourgain-Kontorovich's theorem. Preprint available at arXiv: abs/1207.5168.

[10] И. Д. КАн, Д. А. ФролЕнков. Усиление теоремы Бургейна-Конторовича. Известия РАН. Серия математическая. Том 78, № 2, 2014. Стр. 87-144.

[11] D.A. Frolenkov, I.D. Kan. A strengthening of a theorem of Bourgain-Kontorovich-II. Moskow Journal of Combinatorics and Number Theory, 2014, vol. 4, iss. 1, pp. 78-117

[12] A. A. Dushistova, I. D. Kan, N. G. Moshchevitin. Differentiability of the Minkowski question mark function. J. Math. Anal. Appl. 401, No. 2, $774-$ 794 (2013). 
[13] S. Huang. An improvment on Zaremba's conjecture. Preprint available AT ARXIV:1303.3772v1 [MATH.NT] 14 OCT. 2013.

[14] Р. Вон. Метод Харди - Литтлвуда. М.: Мир, 1985. - 184 с. 